\section{Klimawandel - eine Frage der nationalen Sicherheit?}

\author{
R. McLeman, B. Smit, 2003: Climate \\ Change, Migration and Security. Com- \\ mentary No. 86, a Canadian Security \\ Intelligence Service publication ${ }^{1}$
}

P. Schwartz, D. Randall, 2003: An Abrupt Climate Change Scenario and Its Implications for United States National Security. A report commissioned by the U.S. Defense Department ${ }^{2}$

Anmerkungen zu einer Pentagon-Studie über die Implikationen abrupter Klimaänderungen für die Sicherheit der USA und einer Studie des Kanadischen Security Intelligence Service über Klimawandel, Migration und nationale Sicherheit

\section{von Gerhard Sardemann, ITAS}

Vor drei Jahren, im Juli 2001, während in Bonn die 6. Vertragsstaatenkonferenz zur Klimarahmenkonvention nach einem erfolglosen ersten Anlauf in Den Haag fortgesetzt wurde, erkundigten sich Vertreter des Inselstaates Tuvalu bei den Regierungen Australiens und Neuseelands, ob sie bereit wären, die 11.000 Bewohner Tuvalus aufzunehmen, wenn ihre Inseln wegen des steigenden Meeresspiegels unbewohnbar geworden sein würden. Inzwischen hat sich Neuseeland bereit erklärt, jährlich 75 Bewohner des kleinen pazifischen Inselstaates aufzunehmen.

Was aber wird uns erwarten, wenn dichtbevölkerte Küstenregionen von immer mehr Sturmfluten und Überschwemmungen heimgesucht werden, wenn aufgrund von Dürren die Nahrungsmittelversorgung in weiten Gebieten zusammenbricht, wenn es in anderen Regionen empfindlich kalt wird, weil sich die Zirkulation von Ozean und Atmosphäre verändert? Wird es zu den schon 1990 im Bericht der EnqueteKommission „Vorsorge zum Schutz der Erdatmosphäre“ als die „bedrohlichsten Folgen einer Klimaänderung“ (Enquete-Kommission 1991, S. 334) genannten Wanderungsbewegungen von Umweltflüchtlingen kommen? Diese Fragen stehen im Mittelpunkt zweier Studien, eine davon erstellt von Zukunftsforschern im Auftrag des US-Militärs, die andere verfasst vom kanadischen Security Intelligence Service.
Während die Pentagon-Studie die Problemlage durch Annahme eines Szenarios mit abruptem Klimawandel noch extrem zuspitzt und die Gefahr von mit Massenvernichtungswaffen ausgetragenen Verteilungskonflikten heraufbeschwört, belässt es die kanadische Studie dabei, die Folgen eines anthropogenen Klimawandels abzuschätzen, so wie er vom Intergovernmental Panel on Climate Change (IPCC) in seinem letzten Assessment Report für möglich gehalten wird.

Der Aufsatz „Climate Change, Migration and Security" von Robert McLeman und Barry Smit ist der 86. einer Reihe von Kommentaren des Canadian Security Intelligence Service, in denen seit 1990 langfristige Probleme der kanadischen Sicherheit von internen Spezialisten und von Wissenschaftlern behandelt werden. $\mathrm{Zu}$ den Themen gehören Terrorismus, Proliferation von Waffen, Länderanalysen, aber auch Umwelt und Klima. McLeman und Smit nehmen sich des Themas in unaufgeregter Weise an, wobei sie ihre Argumente durch drei Beispiele veranschaulichen: die „Dust Bowl“Episode in den 30er Jahren in den USA, Wanderungsbewegungen aus den Dürregebieten im östlichen Afrika (Sahel) und die Folgen von Hurrikan „Mitch“, der im Oktober 1998 große Schäden in Honduras und Nicaragua anrichtete.

Selten sind Klimaanomalien allein das auslösende Moment von Wanderungsbewegungen, hinzu kommen schon vorhandene Probleme wie Armut, soziale Konflikte und strukturelle Probleme, die eine Anpassung an sich verändernde Umweltbedingungen verhindern. Andererseits kann die Anpassungsfähigkeit durchaus auch verbessert werden. Nach der katastrophalen Dust Bowl-Episode in den 30er Jahren (Hintergrund übrigens für John Steinbecks Roman „Grapes of Wrath“) kam es zu einer Abwanderung großer Teile der Bevölkerung. Strukturelle Änderungen in der Landwirtschaft, die Schaffung der Möglichkeit zur Bewässerung mit Grundwasser und die Versicherung größerer Risiken haben die Auswirkungen der regelmäßig in den großen Ebenen der USA auftretenden Dürren deutlich verringert. Allerdings waren für derartige Verbesserungen Anstrengungen nötig, für die vor allem die ärmeren Länder, die von einem Klimawandel besonders hart getroffen würden, nicht die nötigen Mittel haben. Aber auch in den USA der 30er Jahre gab es Men- 
schen, die es sich leisten konnten, das Katastrophengebiet zu verlassen, weil sie das Geld hatten, sich anderswo eine neue Existenz aufzubauen oder bei Familienangehörigen in weniger betroffenen Gebieten unterkommen konnten, während andere, aufgrund ihrer begrenzten Mittel meist viel härter von der Dürre Betroffene, dazu keine Möglichkeit hatten.

Gerade das Bestreben, bei Freunden und Verwandten in vermeintlich ,sicheren“ Regionen Unterschlupf zu suchen, lässt die Autoren der Studie auf diejenigen Länder blicken, aus denen schon jetzt die meisten Einwanderer Kanadas kommen: China und Pakistan. Anhand eines Beispiels aus China zeigen die $\mathrm{Au}-$ toren, dass auch eine Verbesserung des Klimas zu einer Verschärfung bestehender gesellschaftlicher Konflikte führen kann: wenn beispielsweise in einem Gebiet die von den bisherigen Bewohnern schon beargwöhnte Zuwanderung anderer Volksgruppen durch eine Erhöhung der Attraktivität wegen besserer Ernten noch verstärkt wird.

Bei entsprechender Infrastruktur könnten auch in Pakistan die von Klimamodellen vorhergesagten zusätzlichen Niederschläge in der Regenzeit zur Bewässerung des Ackerlandes in der Trockenzeit verwendet werden, was zu einer Erhöhung der Erträge der Bauern führen würde. Es ist aber anzunehmen, dass es durch zusätzliche Überschwemmungen und nachfolgende Dürren zu einer weiteren Degradation der Böden und somit zunehmender Landflucht der Bevölkerung kommt. Dies und das hohe Bevölkerungswachstum dürfte die Unruhe in den Städten weiter verschärfen. Auf die Rolle Pakistans für die internationale Sicherheit und im Kampf gegen den Terrorismus wird in dem Aufsatz von McLeman und Smit kurz hingewiesen.

Die Einbeziehung zusätzlicher Risiken durch den Klimawandel in Analysen der nationalen und internationalen Sicherheit sollte aus Sicht der Autoren recht einfach durchzuführen sein, indem man für Regionen, in denen man es sowieso schon mit anderen Risiken zu tun hat und mit einer geringen Anpassungsfähigkeit gegenüber klimabedingten Änderungen rechnen muss, mit Hilfe regionaler Klimamodelle diese Änderungen abzuschätzen versucht. Ein genaueres Bild darüber, wie sich diese Änderungen auswirken, erhält man, indem man untersucht, inwieweit dadurch Ereignisse wie beim Dust-bowl-Phänomen, zyklische Wanderungsbewegungen wie im Sahel oder eine Massenflucht wie nach dem Hurrikan Mitch wahrscheinlich werden. Ein einfaches, aber sicherlich für die Belange von Sicherheitsbehörden ausreichendes Verfahren.

In eine ganz andere Richtung zielt die Studie von Peter Schwartz und Doug Randall für das Pentagon „An Abrupt Climate Change Scenario and Its Implications for United States National Security”. Sie steht unter dem Schlagwort „Imaging the Unthinkable“ und versucht die möglichen Auswirkungen plötzlicher Klimaänderungen auf die nationale Sicherheit der USA abzuschätzen. Dabei geht es nicht darum, die zukünftige Klimaentwicklung genau vorherzusagen, sondern die Intention der Studie geht dahin, „to dramatize the impact climate change could have on society if we are unprepared for it" (S. 7). Dass dies mit Hilfe eines extremen Szenarios getan wird, begründen die Autoren damit, dass ,history tells us that sometimes the extreme cases do occur, there is evidence that it might be and it is DOD's ${ }^{3}$ job to consider such scenarios“ (S. 7).

Plötzliche Klimaänderungen kommen in der Regel „überraschend“; auch in den Berichten des IPCC findet sich der Hinweis auf mögliche Überraschungen, die die Aussagen über Auswirkungen der anthropogenen Treibhausgasemissionen auf das Klima ad absurdum führen könnten. „Inevitable Surprises“ ist der Titel eines Buches von Peter Schwartz (Schwartz 2003), aber auch Untertitel einer Studie der US National Academy of Sciences (NAS) über plötzliche Klimaschwankungen, die im Jahr 2002 erschien und dasselbe Thema behandelte wie die Pentagon-Studie (NAS 2002). Der Begriff „unausweichliche Überraschungen“ impliziert, dass diese Überraschungen durchaus schon voraussehbar waren, man aber trotzdem recht machtlos auf sie zusteuert und die vorausgeahnte Überraschung letztendlich doch als überraschendes Ereignis empfunden wird. Sicher sind auch die Ereignisse des 11. September 2001 in diese Kategorie Überraschungen einzuordnen. Voraussehbar war dieses Ereignis vielleicht sogar im Einzelnen aufgrund von Geheimdienstbeobachtungen; dass irgendetwas passieren könnte, was dem Unbehagen im Gefühl verbreiteter Machtlosigkeit gegenüber der Großmacht USA ein Ventil geben könnte, lag 
ebenfalls ,,in der Luft“ und manifestierte sich in den Anschlägen auf das World Trade Center und das Pentagon. Dass wir es mit einer neuen Sensibilität gegenüber solchen „unliebsamen“ Überraschungen zu tun haben könnten, zeigt auch folgende Einschätzung der Auswirkungen plötzlicher, überraschender Klimaänderungen, die sich in einer Rezension der NAS-Studie über abrupten Klimawandel fand: „Very important, perhaps more so in the long run than human terrorism, which has elicited a robust response.“

Das von Schwartz und Randall entwickelte Szenario basiert auf einem Ereignis in der Klimageschichte vor etwa 8.200 Jahren, über das in der NAS-Studie folgender Satz zu lesen war: „The event punctuated generally warm conditions not too different from recently, so warmth is not a guarantee of climate stability." (NAS 2002, S. 29). Man nimmt an, dass es nach einem Anstieg der Temperaturen und abschmelzenden Gletschern zu einer erhöhten Süßwasserzufuhr im Nordatlantik und einer nachfolgenden Abschwächung der thermohalinen Zirkulation im Ozean kam. Schon 1985 hatte das Wallace Broecker zur Erklärung der rapiden Abkühlung nördlicher Breiten während des Jüngeren Dryas vor 12.000 Jahren vorgeschlagen. Überhaupt scheint die sich doch recht schnell in der Vorstellung vieler Menschen einnistende, weil einfache und vor allem bildhafte, Theorie vom abgeschalteten ,ozeanischen Förderband“" (conveyor belt) zyklisch immer wieder in den Vordergrund geschwemmt zu werden. In ihrer ursprünglichen Fassung ließ sich die Theorie allerdings nicht halten und man witzelte, dass Broecker schon bald eine noch elegantere Hypothese - ,an even more elegant hypothesis“ - vorstellen würde, was er 1990 auch tat (Jones 1991). Kurz darauf war über den Golfstrom in „bild der wissenschaft“ zu lesen: „Diese Jahrtausende alte Warmwasserheizung droht nun binnen weniger Jahrzehnte zusammenzubrechen“ (Weiner 1991). Aber auch Schwartz glaubt nun, wie er auch in einer Präsentation seines Buchs „Inevitable Surprises“ (Schwartz 2003) schreibt, mit der beobachteten Abnahme des Salzgehaltes im Nordatlantik tatsächlich ein erstes Indiz dafür gefunden zu haben, dass uns schon bald eine derartige Überraschung erwarten könnte.

Das Ereignis vor 8.200 Jahren führte ebenfalls zu einer Abkühlung in den nördlichen
Breiten, allerdings nicht in der Intensität wie während des Jüngeren Dryas. Wie sich heute ein abrupter Klimawandel wie der vor 8.200 Jahren auf das regionale Klima auswirken könnte, zeigt der „Wetterbericht“ in der Studie von Schwartz und Randall (S. 10):

- lang anhaltende Dürren in landwirtschaftlich wichtigen Gebieten Europas und im Osten Nordamerikas,

- Absinken der mittleren Temperaturen um $2,8^{\circ} \mathrm{C}$ über Asien und Nordamerika und bis zu $3,3^{\circ} \mathrm{C}$ über Europa,

- Temperaturanstieg um bis zu $2,2^{\circ} \mathrm{C}$ über Australien, Südamerika und Südafrika,

- Intensivierung der Winterstürme.

Gegen die Annahme, dass sich Ereignisse wiederholen können, die in der jüngeren Klimageschichte schon mehrfach, allerdings in unterschiedlicher Ausprägung, vorkamen, ist sicherlich nichts einzuwenden. Dass wir gerade jetzt auf ein solches Ereignis zusteuern sollen, ist aber wohl eher der Absicht der Autoren geschuldet, ein wirkungsvolles Szenario zu konstruieren. Was das Verhalten der Menschen angeht, die einer derart katastrophalen Änderung des Weltklimas gegenüber stehen, so wird auch hier der Rückfall in archaische Verhaltensmuster als gegeben angenommen. Hungern oder ums Brot kämpfen („to raid“) wird als einzige Alternative vorgegeben und der Mensch wird sich dann wohl fürs Kämpfen entscheiden, was natürlich die Studie erst für das Pentagon interessant macht. Man fragt sich unwillkürlich, welche Bezeichnung man sich für Staaten einfallen lassen wird, die aufgrund der in der Studie beschriebenen widrigen Umstände ums nackte Überleben kämpfen werden - insbesondere, wenn die anderen Staaten „die Schotten dicht machen“. Vielleicht bleibt man beim bislang üblichen Begriff „Schurkenstaaten“.

Dass man nun die Möglichkeit eines plötzlichen Klimawandels und seine katastrophalen Folgen derart in den Vordergrund stellt wie es in den USA gerade geschieht, lenkt natürlich von den Auswirkungen menschengemachter Klimaänderungen $a b$, stellt diese förmlich in den Schatten - auch wenn eine anthropogene Klimaerwärmung durchaus der Auslöser für einen solchen abrupten Wandel darstellen kann. Was aber sollen Vereinbarungen zur Reduktion von Treibhausgasemissionen wie das Kiotoprotokoll, wenn wir uns für Katastro- 
phen rüsten müssen, die durch abrupte Klimaänderungen hervorgerufen werden? Man sieht förmlich die Schlote rauchen in Vorbereitung auf dieses Ereignis.

Abgesehen von derartigen Einwänden ist die Beschäftigung mit der Möglichkeit abrupten Klimawandels durchaus sinnvoll und angebracht. Die Studie von Schwartz und Randall liest sich zudem recht spannend, auch wenn sie teilweise stark an das Drehbuch zu einem Katastrophenfilm erinnert. Das mag den SWR in einer Ankündigung für eine Diskussionssendung über Roland Emmerichs Film „The Day after Tomorrow“ dazu verleitet haben, die Studie als Vorlage für diesen Film zu bezeichnen. Denjenigen, die sich etwas fundierter mit dem Thema „Abrupter Klimawandel und seine ökologischen und ökonomischen Folgen“ auseinandersetzen wollen, sei zusätzlich die NAS-Studie anempfohlen, die allerdings mit 244 Seiten anstatt der 22 an die Öffentlichkeit gelangten Seiten der Pentagon-Studie daherkommt. Nach dem Aufsehen, das die Pentagonstudie und vor allem der Film „The Day after Tomorrow“ auch in Zeitschriften wie „Science“ und „Nature“ erregten, erschien jedoch im Mai 2004 eine vierseitige Kurzfassung der NAS-Studie.

\section{Anmerkungen}

1) http://www.csis-scrs.gc.ca/eng/comment/ com86_e.html

2) http://www.ems.org/climate/pentagon_ climatechange.pdf

3) U.S. Department of Defense

\section{Literatur}

Enquete-Kommission „Vorsorge zum Schutz der Erdatmosphäre“ (Hrsg.), 1991: Schutz der Erde. Teilband 1. Bonn, Karlsruhe: Economica Verlag, C.F. Müller

Jones, G.A., 1991: A stop-start ocean conveyer. In: Nature, 349, S. 364-365

NAS/National Academy of Sciences, 2002: Abrupt Climate Change: Inevitable Surprises. A study of the Committee on Abrupt Climate Change. National Research Council, Washington, D.C.: National Academy Press; http://www.nap.edu/catalog/10136.html; Kurzfassung, 2004: http://www.nap.edu/html/ climatechange-brief/abruptclimatechange-brief.pdf

Schwartz, P., 2003: Inevitable Surprises: Thinking Ahead in a Time of Turbulence. Gotham, New York; http://www.gbn.org/ArticleDisplayServlet.srv?aid= 14200

Weiner, M., 2001: Wenn der Golfstrom stockt. bild der wissenschaft 1 , S. 16-20

\section{N.C. KARAFYLLIS (Hrsg.): Biofakte. Versuch über den Menschen zwischen Artefakt und Lebewesen. Paderborn: mentis Verlag, 2003, 295 S., ISBN 3-89785-384-1, Euro 32,00}

\section{Rezension von Gerhard Banse, ITAS}

Hintergrund bzw. Ausgangspunkt des vorliegenden Buches ist die philosophische Anthropologie (,Versuch über den Menschen“), die gegenwärtig verstärkt den Menschen als „Hybrid“ (Latour) zwischen Natur- und techniknutzendes Wesen thematisiert. Techniknutzung erweist sich dabei nicht nur als dem Mensch „äußerlich“, sondern erfasst ihn auch „innerlich“, und zwar nicht nur in der bekannten Weise, dass seine „Wesenskräfte“ (Wissen, Erfahrungen, Fähigkeiten, Fertigkeiten, Praxen) verändert werden, sondern dass zunehmend auch seine „Körperlichkeit“ Gegenstand bzw. Ort der Techniknutzung ist, zielgerichtet beeinflusst, verändert, manipuliert, technisiert. Produkt dieser Entwicklung sind „Biofakte“, eine Begrifflichkeit, die die Herausgeberin des vorliegenden Buches kreiert hat, begrifflich gefasst als Verschmelzung von „Leben“ (griech. bios) und „Artefakt“ (= Künstliches). Ein Biofakt ist somit ein semiartifizielles Lebewesen, etwas „zwischen“ - im Sinne der klassischen Auffassung dem von sich aus „Gewachsenen“ bzw. „Gewordenen“ (= Natur einschließlich des Menschen) und dem „Hergestellten“, „Gemachten“ (= Artefakte einschließlich der Technik). Vor allem die aktuellen bio-, informations- und nanotechnischen Entwicklungen bergen ein entsprechendes Potenzial in sich, diese Trennung zu überwinden, eine strikte Unterscheidung von „Natürlichkeit“ und „Künstlichkeit“ als problematisch erscheinen zu lassen. Die über gentechnologische Verfahren ermöglichte technische Manipulation der „Ursprungs-“ oder Ausgangsbedingungen des Lebens belegt das mit aller Deutlichkeit. Aber es sind nicht nur - oder vor allem? - diese in der Öffentlichkeit viel und kontrovers debattierten Realitäten. „Biofakti- 